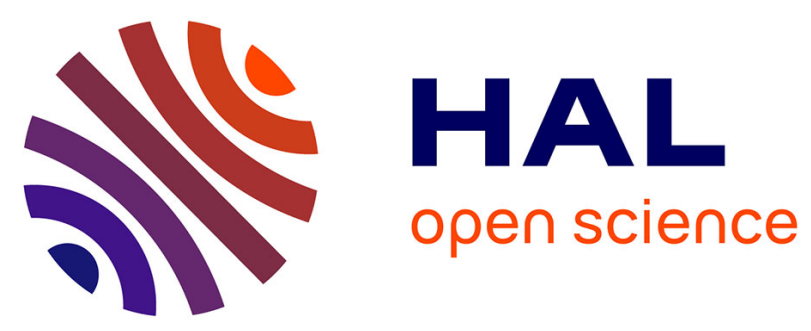

\title{
Transient over-expression of estrogen receptor- $\alpha$ in breast cancer cells promotes cell survival and estrogen-independent growth
}

Robert S. Tolhurst, Ross S. Thomas, Fiona J. Kyle, Hetal Patel, Manikandan Periyasamy, Andrew Photiou, Paul T. R. Thiruchelvam, Chun-Fui Lai, Marwa Al-Sabbagh, Rosemary A. Fisher, et al.

\section{To cite this version:}

Robert S. Tolhurst, Ross S. Thomas, Fiona J. Kyle, Hetal Patel, Manikandan Periyasamy, et al.. Transient over-expression of estrogen receptor- $\alpha$ in breast cancer cells promotes cell survival and estrogen-independent growth. Breast Cancer Research and Treatment, 2010, 128 (2), pp.357-368. 10.1007/s10549-010-1122-6 . hal-00615373

\section{HAL Id: hal-00615373 https://hal.science/hal-00615373}

Submitted on 19 Aug 2011

HAL is a multi-disciplinary open access archive for the deposit and dissemination of scientific research documents, whether they are published or not. The documents may come from teaching and research institutions in France or abroad, or from public or private research centers.
L'archive ouverte pluridisciplinaire HAL, est destinée au dépôt et à la diffusion de documents scientifiques de niveau recherche, publiés ou non, émanant des établissements d'enseignement et de recherche français ou étrangers, des laboratoires publics ou privés. 
Transient Over-expression of Estrogen Receptor- $\alpha$ in Breast Cancer Cells Promotes Cell Survival and Estrogen Independent Growth

Robert S. Tolhurst ${ }^{1}$, Ross S. Thomas ${ }^{1}$, Fiona J. Kyle, Hetal Patel, Manikandan Periyasamy, Andrew Photiou, Paul T. R. Thiruchelvam, Chun-Fui Lai, Marwa Alsabbagh, Rosemary A. Fisher, Sayka Barry*, Tatjana Crnogorac-Jurcevic*, Lesley-Ann Martin\#, Mitch Dowsett" ${ }^{\#}$, R. Charles Coombes, Tahereh Kamalati, Simak Ali and Laki Buluwela

${ }^{1}$ These authors should be considered as joint first authors.

Division of Cancer, Department of Surgery \& Cancer, Imperial College London, Hammersmith Hospital Campus, Du Cane Road, London W12 ONN, UK.

${ }^{*}$ Centre for Molecular Oncology \& Imaging, Barts and The London School of Medicine and Dentistry, Queen Mary University of London, Institute of Cancer, Charterhouse Square, London EC1M 6BQ, UK.

"Breakthrough Breast Cancer Centre, Institute of Cancer Research, London, SW3 6JJ, UK

ףThe Royal Marsden Hospital, London, SW3 6JB UK

The authors declare no conflict of interest.

Address correspondence to: S. Ali or L. Buluwela, Division of Cancer, Department of Surgery \& Cancer, Imperial College London, Hammersmith Hospital Campus, Du Cane Road, London W12 ONN, UK; email: simak.ali@imperial.ac.uk; I.buluwela@imperial.ac.uk 


\section{Abstract}

Estrogen receptor- $\alpha(E R \alpha)$ positive breast cancer frequently responds to inhibitors of ERa activity, such as tamoxifen, and/or to aromatase inhibitors that block estrogen biosynthesis. However, many patients become resistant to these agents through mechanisms that remain unclear. Previous studies have shown that expression of ERa in ERa-negative breast cancer cell lines frequently inhibits their growth. In order to determine the consequence of ERa overexpression in ERa-positive breast cancer cells, we over-expressed ERa in the MCF-7 breast cancer cell line using adenovirus gene transduction. ER $\alpha$ overexpression led to ligand-independent expression of the estrogen-regulated genes pS2 and PR and growth in the absence of estrogen. Interestingly, prolonged culturing of these cells in estrogen-free conditions led to the outgrowth of cells capable of growth in cultures from ERa transduced, but not in control cultures. From these cultures a line, MLET5, was established which remained ERapositive, but grew in an estrogen-independent manner. Moreover, MLET5 cells were inhibited by anti-estrogens showing that ERa remains important for their growth. Gene expression microarray analysis comparing MCF-7 cells with MLET5 highlighted apoptosis as a major functional grouping that is altered in MLET5 cells, such that cell survival would be favoured. This conclusion was further substantiated by the demonstration that MLET5 show resistance to etoposide induced apoptosis. As the gene expression microarray analysis also shows that the apoptosis gene set differentially expressed in MLET5 is enriched for estrogen-regulated genes, our findings suggest that transient over-expression of ERa could lead to increased cell survival and the development of estrogenindependent growth, thereby contributing to resistance to endocrine therapies in breast cancer patients. 


\section{Introduction}

Two-thirds of breast cancers express estrogen receptor- $\alpha$ (ER $\alpha$ ), and estrogen plays a critical role in the development and progression of these tumours. This understanding has led to the development of anti-estrogens, primarily tamoxifen, which compete with estrogen for binding to the ERa. Treatment with tamoxifen for 5 years following surgery leads to $50 \%$ lower annual recurrence rate and a $28 \%$ decrease in annual rates of mortality in patients with early stage ER-positive breast cancer [1, 2]. However, many patients who respond to tamoxifen, eventually relapse. Aromatase inhibitors act by preventing the conversion of androgens into estrogens by the aromatase enzyme, with new aromatase inhibitors displaying greater efficacy than tamoxifen. However, resistance to aromatase inhibitors also develops in many cases [3,4].

In a proportion of cases, patients who initially present with ERa-positive breast cancer, become ERa-negative [5]. The mechanisms by which ERa expression is lost are unclear, although epigenetic silencing of the ERa gene may be involved [6]. In most cases, however, resistant tumours remain ERapositive and show a response to a change of endocrine agent [2], indicating that $E R \alpha$ continues to be important in regulating tumour growth in these cases. For the latter, recent studies suggest that endocrine resistance could result from modulation of ERa activity by altered co-activator and co-repressor balance and/or crosstalk with growth factor signalling cascades, including phosphorylation of ER $\alpha$ at specific residues. In this context, elevated HER2 and EGFR expression have been observed in cell line models of tamoxifen resistance, whilst elevated ERK1/2 MAPK [7] and high levels of phosphorylated AKT have been associated with poor response to tamoxifen and a worse patient prognosis [8]. Further, phosphorylation of ERa at serine 118 (S118) is elevated in recurrence following tamoxifen treatment [9]. Finally, high-level expression of the coactivator AIB1 is associated with poor response to tamoxifen in ERa-positive breast cancer, with AIB1- and HER2-positive patients having the worst outcome following tamoxifen treatment [10]. 
Ectopic expression of ERa in ERa-negative breast cancer cell lines and in immortalised non-tumourigenic breast cells inhibits their growth, despite showing estrogen-dependent stimulation of expression of estrogen-responsive genes [1113]. By contrast, over-expression of ERa did not inhibit the growth of ERapositive breast cancer cell lines [12], although conditional over-expression of ERa did lead to increased growth of MCF-7 cells in the absence of ligand [14], suggesting that ERa over-expression in ERa-positive breast cancer cells may facilitate adaptation to estrogen deprivation.

To further investigate the consequences of ER $\alpha$ over-expression on the estrogen responses in ER $\alpha$-positive breast cancer cells, we have transduced MCF-7 cells with an adenovirus encoding ERa. Prolonged culturing of the ER $\alpha-$ transduced cells in estrogen-free medium allowed the establishment of an estrogen-independent line, MLET5, in which ERa expression was maintained. MLET5 cells did not retain adenoviral sequences and microsatellite genotyping confirmed their lineage as MCF-7-derived. In further characterising MLET5 cells by gene expression microarray analysis, we found changes in apoptosis associated gene expression when compared to MCF-7 cells, so as to favour cell survival. As a result of these differences, we have gone on to show that the MLET5 line has altered cell survival characteristics, as indicated by a greatly reduced sensitivity to etoposide induced apoptosis. Together, these findings indicate that transient ER $\alpha$ over-expression in breast cancer cells could be sufficient to promote the development of endocrine resistance in breast cancer through altered expression of estrogen-regulated genes involved in cell survival. 


\section{Materials and Methods}

\section{Cell culture and cell lines}

MCF-7 cells were obtained from the ATCC (LGC Prochem, USA) and maintained in Dulbecco's modified Eagle's medium (DMEM) (Sigma-Aldrich Ltd., UK) supplemented with $10 \%$ fetal calf serum (FCS) (First Link Ltd., UK). For culturing in estrogen-free conditions, MCF-7 cells were cultured in DMEM lacking phenol red (DMEM-PR) (Gibco-BRL, UK), supplemented with 10\% dextrancoated charcoal-stripped FCS (DSS) (First Link Ltd., UK). MLET5 cells were routinely cultured in DMEM-PR, containing 10\% DSS.

Recombinant adenovirus construction and infection of MCF-7 cells

The human ERa open reading frame was cloned into the adenoviral shuttle vector pAdTrack-CMV [15], which encodes GFP, to generate pAdTrackCMV-ERa. Recombinant adenoviral genome AdERa and control virus (AdGFP) were generated following recombination by co-transformation of $E$. coli BJ5183 cells with pAdEasy-1, as described and packaged in HEK293 cells, also as described [15]. The viruses were purified by caesium chloride banding and viral particle concentration was determined by spectrophotometric analysis. MCF-7 cells $\left(6 \times 10^{6}\right)$ were seeded in $10-\mathrm{cm}$ plates in DMEM-PR, containing $10 \%$ DSS and allowed to settle for 24 hours prior to infection. FACS analysis of single cell suspensions prepared 2 days following adenoviral transduction, was used to determine the percentage of cells transduced. Cell counts were performed using a haemocytometer with trypan blue exclusion for counting of viable cells.

Sulphorhodamine B (SRB) Growth assay

Sixteen hours following seeding of $3 \times 10^{3}$ cells in 96-well plates in DMEMPR containing 10\% DSS, the medium was replaced with fresh medium supplemented with 17ß-estradiol (E2), anti-estrogens, or an equivalent volume of the vehicle (ethanol). Medium was changed every three days. Cells were fixed using $40 \%(\mathrm{w} / \mathrm{v}) \mathrm{TCA}$, for one hour at $4^{\circ} \mathrm{C}$, washed five times with distilled, 
deionised $\mathrm{H}_{2} \mathrm{O}$, followed by incubation with $0.4 \%$ SRB in $1 \%$ acetic acid for one hour at room temperature. Excess dye was removed with five washes with 1\% acetic acid and drying at room temperature. Absorbance at $480 \mathrm{~nm}$ was determined following solubilisation of the dye by the addition of $100 \mu \mathrm{l}$ of $10 \mathrm{mM}$ Tris base to each well.

For measuring growth following addition of Etoposide (Sigma-Aldrich), $2 \times 10^{3}$ cells were seeded in each well. Medium supplemented with the inhibitors in a titration of two-fold dilutions, starting from $100 \mu \mathrm{M}$, was added after 48 hours. Cell growth was measured 48 and 72 hours after treatment, using the SRB assay, with the $\mathrm{GI}_{50}$ being defined as the concentration of drug required to obtain $50 \%$ of the growth exhibited by untreated, control cells.

\section{Cell cycle analysis}

Cells were seeded in six-well plates (10 $/$ well) in DMEM containing $10 \%$ FCS and allowed to adhere for $48 \mathrm{~h}$, followed by the addition of $1 \mathrm{nM}-100 \mathrm{M}$ etoposide (Sigma-Aldrich) or DMSO and incubation for $48 \mathrm{~h}$. Cells were harvested, cell cycle Annexin V/propidium iodide staining and analysis was carried out as previously described [16].

\section{$R T-P C R$ analysis}

RNA was prepared and RT-PCR performed as described previously [17]. For quantitative RT-PCR (Q-RT-PCR) measurements, Taqman Gene Expression Assays were used with a $7900 \mathrm{HT}$ Fast Real-time PCR machine (Applied Biosystems). Primer details are given in supplementary information.

\section{Immunoblotting}

MCF-7 and MLET cells $\left(1 \times 10^{6}\right)$ were seeded in $10-\mathrm{cm}$ plates DMEM containing $10 \%$ FCS and lysed after 48 hours, as described [17]. For experiments where ligands were to be added, the cells were incubated in DMEM-PR containing $10 \%$ DSS for three days prior to seeding. Ligands were added, as appropriate, with an equal volume of ethanol being added to the controls. Cell lysates were prepared 
24 hours later. Immunoblotting was carried out as described previously [17], using antibodies detailed in Supplementary Material.

Gene expression microarray analysis

For gene expression microarray analysis, MCF-7 and MLET5 cultures were seeded in estrogen-depleted medium as described above for 3 days, with three bioreplicate cultures used for each cell line and treatment. Following 16 hours treatment with 10nM E2, RNA was purified from the cultures (RNeasy, Quiagen) and used to probe bead arrays (Illumina, Human WG-6) through Cambridge Genomic Services (http://www.path.cam.ac.uk/cgs/). Hybridisation data were obtained using BeadStudio software (Illumina) and raw gene expression data analysed using GeneSpring GX 10 software (Agilent, Santa Clara USA). All samples were normalised by quantile normalisation to minimise variation between microarray chips. Data were filtered to include only those probes expressed in at least one sample (present and marginal flags). The three replicates for the no ligand and E2 treatments were compared by unpaired $t$ tests, and differentially expressed genes were considered significant at a multiple testing corrected $p$ value (Benjamini Hochberg FDR) of $<0.05$. 


\section{Results}

Adenoviral transduction of ERa stimulates MCF-7 cell growth and expression of estrogen-regulated genes

MCF-7 cells were infected with an adenovirus encoding GFP for monitoring infection (AdGFP), into which the human ERa coding region was inserted (AdER $\alpha$ ) (Supplementary Fig. 1). FACS analysis showed that 47\%, 76\% and $90 \%$ of the cells were GFP-positive using multiplicity of infection (MOI) of 400, 2,000 and 10,000 particles per cell, respectively. However, infection with AdGFP at $\mathrm{MOI}=10,000$ resulted in cell death, so all subsequent infections were performed using $\mathrm{MOI}=2,000$. Under these conditions, stimulation of growth was observed in the absence as well as in the presence of estrogen. ERa levels were 5-6-fold higher in the AdERa-infected cells, compared to the AdGFP-infected cells at the protein and RNA levels (Fig. 1A, B). However, in the presence of estrogen, expression of the estrogen-responsive PR and pS2 genes was similar in both cases (Fig. 1B-E), with inhibition of expression with the anti-estrogen ICI182,780 (ICI), but their expression was markedly elevated in the absence of ligand, suggesting that adenoviral over-expression of ERa leads to ligandindependent activation of E2-regulated genes, as well as growth in the absence of estrogen.

Survival of MCF-7 cells transduced with ERa is enhanced during long-term culturing in estrogen-depleted medium

Since transduction of ERa in MCF-7 cells conferred a growth advantage in estrogen-free culture conditions, we determined the effect of long-term culturing of AdERa-infected MCF-7 cells in E2-depleted growth medium (Fig. 1F). Under these conditions, cell numbers fell in all cultures over a period of 2-3 months, resulting in a complete loss in the mock and AdGFP-infected cultures by month 5. By contrast, cell numbers started to recover in the AdERa cultures. The loss of control AdGFP infected and mock infected MCF-7 cells, with the emergence of a small number of surviving cells following infection with AdERa over the course of 5-6 months, was observed in four additional, independent transduction 
experiments. In order to confirm these findings, the infections were repeated. From 3 out of the 5 separate infections and selection over 8 months, the recovered cells were cultured, one of these giving rise to the MLET5 cell line. Monitoring of the cultures by fluorescence microscopy showed no evidence of GFP expression 2-3 months following infection; nor was GFP expression evident in the MLET5 line (Supplementary Fig. 1E). Furthermore, there was no detectable expression of GFP by RT-PCR, nor was there any evidence for the presence of adenoviral sequences by PCR (data not shown). These observations are consistent with adenoviruses being non-integrative and, as a result of cell division, having transduced genomes that become diluted, resulting in loss of transgene expression. Finally, microsatellite genotyping analysis showed that MLET5 cells are indeed identical to MCF-7 cells for 10 markers examined (Table 1). All of the markers used are highly polymorphic with multiple alleles. Assuming a conservative figure of 0.5 for the frequency of each allele at the ten loci examined, the probability that the cell lines are not derived from MCF-7 is $0.5^{16}$ $(p<0.0001)$ (Supplementary Table 1).

MLET5 cells grow in an estrogen-independent manner, but show differential sensitivity to anti-estrogens

Estrogen is required for MCF-7 cell growth (Fig. 2A). By contrast, growth of the MLET5 cells was independent of E2, and was indistinguishable from growth in the absence of ligand (Fig. 2B). Moreover, in contrast to MCF-7 cells, the growth of MLET5 cells was only partially inhibited by anti-estrogens, (Fig. 2CD). At the RNA level ER remained unchanged in MLET5 cells, although there was an apparent decrease in ER expression in the absence of E2 (Fig. 3A). Overall however, ER protein levels were elevated in MLET5 cells compared with MCF-7 cells (Fig. $3 \mathrm{H}$ ), which may be indicative of a post-transcriptional mechanism of ER protein stabilisation in MLET5 cells. Despite the elevated level of ER protein, expression of several well-characterised estrogen-regulated genes was reduced (PR, CTD, TFF1/pS2), at the mRNA and protein level (Fig. 
3B-D and Fig. 3H), suggesting that the responsiveness of some, but not all E2regulated genes (GREB1, MYC, CCND1; Fig. 3F, G, H) is attenuated in MLET5 cells.

This reduced stimulation in expression of some estrogen-regulated genes could be due to a reduction in ER phosphorylation in MLET5 cells. However, levels of Ser118 and Ser167 phosphorylation (Supplementary Fig. 2), previously associated with increased ER activity were found to be higher in MLET5 cells than in MCF-7 cells, with the higher levels of phosphorylation reflecting the elevated ER expression in MLET5 cells. Alternatively, altered ER transcriptional co-regulator levels could lead to attenuation of expression of regulated genes [18-20]. Although expression of the p160 co-activators of ER SRC1 and AIB1 were not altered in MLET5 cells (Supplementary Fig. 3), there was a marked upregulation of the co-repressor SMRT in MLET5 cells, suggesting the possibility of its involvement in reduced expression of E2-responsive genes in MLET cells.

\section{Gene expression microarray analysis of MLET5}

In order to determine if reduced estrogen regulation was a general feature of ER activity in MLET5 cells, and to determine the gene expression changes that may have led to the development of the estrogen-independent outgrowth of MLET5 cells, gene expression microarray analysis was carried out. For this, RNA prepared from MCF-7 and MLET5 cells cultured in estrogen-depleted medium, followed by addition of E2 for 16 hours, was used to probe Illumina Human WG-6 bead chips. Of the 42,620 genes on the array, 4,353 genes showed significant differential expression in MCF-7 in the presence of E2, compared with the vehicle control, with similar numbers of genes showing up-and down-regulation. About half as many $(2,134)$ genes showed significant differential expression in MLET5 cells, consistent with the reduced expression of estrogen-regulated genes observed using Q-RT-PCR (Fig. 3). Applying a fold change (FC) of 1.5 showed that 414 and 971 genes were up-, or down-regulated with estrogen in MLET5 and MCF-7 cells, respectively (Fig. 4A, B). Of the genes showing 1.5-fold upregulation in expression in E2-treated MCF-7 cells, 34\% (171 of 501) genes were 
also up-regulated $\geq 1.5$-fold in MLET5 cells (representing $79 \%$ of the genes upregulated by estrogen) and included known estrogen regulated targets such as GREB1, PDZK1, PGR, MYB, RET and IGFBP4 (Supplementary Table 2). It should also be noted that a further 140 genes with $\geq 1.5$ fold induction by estrogen in MCF-7 cells showed a significant estrogen regulation in MLET5 cells perse.

Similarly, $115(24 \%)$ of the estrogen down-regulated genes in MCF-7 cells were also down-regulated $>1.5$ fold in MLET5 and a further 140 genes showed significant down-regulation in MLET5 cells. These data are shown in a scatterplot analysis (Fig. 4C) and highlights the fact that most of the genes that show estrogen regulation in MCF-7 cells are also estrogen-regulated in MLET5 cells, indicating that despite their adaptation to growth in the absence of estrogen, MLET5 cells still feature a marked, but attenuated estrogen-regulated gene response.

In examining the MCF-7 and MLET5 gene expression profiles we also noted that a number of previously described estrogen up-regulated genes which showed moderate, but significant estrogen regulation in MCF-7 cells, were upregulated in MLET5 cells in the absence of ligand. For example, the expression of CXCL12 was 4.4 fold higher in MLET5 than in MCF-7. The same was also true for some estrogen down-regulated genes, such as INHBB, whose expression was 0.25 fold in vehicle treated MLET5 cells compared to MCF-7 cells. This suggests the possibility that in MLET5 cells ER regulates the expression of estrogen responsive genes in the absence of estrogen and that estrogen is able to further modulate the expression of these genes.

\section{Estrogen regulation and apoptosis in MCF-7 and MLET5 cells}

A total of 717 genes were differentially expressed $(p<0.05, F C \geq 2.0)$ in MLET5 compared with MCF-7, whilst 886 genes showed differential expression in the presence of estrogen, with 563 of these genes being differentially regulated in both the vehicle and estrogen-treated samples (Fig 4D). Pathway analysis of the genes showing differential expression in MLET5 cells compared with MCF-7 
cells ( $p<0.05, F C \geq 2.0$ ) using gene set enrichment analysis (GSEA) identified cellular and molecular functional groupings (Supplementary Table 3), the most significant of which were functional groups encompassing apoptosis and included molecules involved in cell survival and the regulation of cell death. David (http://david.abcc.ncifcrf.gov/) also showed significant enrichment of cell death groups in MLET5 cells (Supplementary table 3).

With GSEA 5 of the top 6 annotated groups are associated with apoptosis and accounted for 22 genes showing differential expression in MLET5 v MCF-7 cells, including the key apoptotic regulators BCL2, BAD and BIK (Table 1; Supplementary Fig. 4). Q-RT-PCR showed that BCL2 expression was estrogen regulated in MCF-7 and MLET5 cells, but was dramatically elevated in MLET5 cells (Fig. 5A). Conversely, the BH3-only pro-apoptotic BIK (BCL2-interacting killer) gene was down-regulated by estrogen in MCF-7 and MLET5 cells and its expression was greatly reduced in MLET5 cells (Fig. 5B). The related gene BID, which showed estrogen-regulation, was also down-regulated in MLET5 cells (Fig. $5 C$ ), whereas expression of $B A D$, reduced upon estrogen treatment in MCF-7 cells, was increased in MLET5 cells (Fig. 5D). Western blotting confirmed Q-RTPCR data, with reduced BID and BIK and elevated BCL2 and BAD (Fig. 5E).

These findings suggest that altered cell survival is a key feature in the progression of the estrogen-independent MLET5 cells. To test this, the response of MCF-7 and MLET5 cells to the pro-apoptotic drug, etoposide was compared. MLET5 cells were considerably less sensitive to etoposide, with a $\mathrm{Gl}_{50}=19.3$ $\mu \mathrm{M}$, compared with a $\mathrm{GI}_{50}=4.6 \mu \mathrm{M}$ for MCF-7 (t-test: $\mathrm{p}<0.0001$ ) (Fig. 6A, B). Cell cycle analysis performed with etoposide at concentrations ranging from $1 \mathrm{nM}$ $100 \mu \mathrm{M}$ showed an accumulation of MCF-7 and MLET5 in G2/M (Fig. 6C). There was also an accumulation of MCF-7 cells in the sub-G1 phase, indicative of cells undergoing apoptosis, which was confirmed with direct determination of cells in apoptosis following staining for Annexin $\mathrm{V}$, with $60 \%$ of the MCF-7 cells undergoing apoptosis in the presence of $100 \mu \mathrm{M}$ etoposide (Fig. 6D). However, even at a concentration of $100 \mu \mathrm{M}$, the proportion of MLET5 cells in apoptosis is similar to that seen for vehicle (DMSO) treated cells. 


\section{Discussion}

Estrogen Receptor- $\alpha$ over-expression by adenoviral transduction results in ligand-independence in MCF-7 cells

In the normal breast, ERa expression is restricted to a small proportion of epithelial cells, with ERa expression being associated with a lack of estrogen regulated proliferation. In the majority of primary breast cancer patients, however, the cancer cells are distinguished by expression of ERa in proliferating cells, with levels of expression often being elevated. Although the presence of ERa in breast cancer is associated with the likelihood of response to endocrine therapies, the impact of ERa levels in breast cancer cells on prognosis and response to endocrine treatments remain unclear. Determination of ERa levels using a ligand binding assay showed that patients with high ERa levels had a shorter relapse-free period than patients with low ERa [21]. Further, fluorescence in situ hybridization of ERa-positive breast tumors has shown that the ERa gene (ESR1) is amplified in about $20 \%$ of cases, the amplification being strongly associated with poor disease-free survival [22], whilst multiplex ligationdependent probe amplification-based copy number analysis shows a similar percentage of ESR1 amplification in breast cancer, an association with higher mitotic index and a trend towards higher grade [23]. Other studies have shown similar levels of ER $\alpha$ gene amplification, but by contrast, ESR1 amplification was associated with better response to endocrine therapies [24, 25]. Further adding to the controversy are findings from other laboratories showing that the frequency of ESR1 amplification is considerably lower than 20\% [26]. Additionally, there is no apparent relationship between ERa levels and response in patients with advanced disease; however, and response in the IMPACT trial which assessed tamoxifen, anastrozole or the combination in the neo-adjuvant setting, had significantly more responders in the patient group with higher ERa levels, but only in the combination arm [27]. Therefore, it remains unclear as to whether ERa levels are related to clinical response.

In order to better understand the consequences of high ER $\alpha$ expression in breast cancer cells, we over-expressed ERa in the MCF-7 ERa-positive breast 
cancer cell line. Interestingly, the transient over-expression of ERa led to the eventual outgrowth of cells that were estrogen-independent for growth. These cells were negative for GFP expression and PCR indicated that adenoviral sequences are absent from these lines. Further, in the generation of LTED cells, [28] noted a transient over-expression of ERa levels in MCF-7 cells within three weeks of culturing in an estrogen deprived environment. This transient overexpression preceded susbsequent changes leading to estrogen independent growth, in which ERa over-expression was again seen. Taken together, these findings suggest that transient over-expression of ERa could play a role in the acquisition of an estrogen-independent phenotype by breast cancer cells. The mechanisms by which ERa over-expression may promote the development of resistant cells are unclear, but one possibility is that transient ERa overexpression provides a survival advantage that allows estrogen-independent cells to emerge. The minimum period of ERa over-expression that would be sufficient for establishment of the resistant lines is also unclear. However, at an MOI of 2,000 particles/cell, $90 \%$ of the cells were GFP-positive on day 3 following infection, falling to $48 \%$ on day 9 and no GFP expression was detectable after 12 weeks in culture (data not shown; and see Fig. 1F). Hence continued overexpression of transduced ERa was not required for the emergence of the MLET lines.

The MLET5 line was not responsive to estrogen for growth but was partially sensitive to anti-estrogens, indicating the continued importance of ERa in MLET5. Although ERa protein levels were significantly higher in MLET5 compared to MCF-7 cells, E2 induction of pS2 and CTD was markedly reduced. Phosphorylation of ER $\alpha$ at $\mathrm{S} 118$ and $\mathrm{S} 167$ stimulates its activity [29] and phosphorylation at these sites was seen to be increased in MLET5, suggesting that the reduced apparent ERa activity is not due to a reduction in ERa phosphorylation. However, levels of NCoR and particularly SMRT were elevated in MLET5 cells, compared to MCF-7 cells. This is in contrast to a previously described MCF-7 derived line, LCC1, generated through in vivo selection under conditions of low estrogen availability, and LCC9, derived from LCC1 following 
selection for growth in the presence of ICI [30]. LCC1 and LCC9 remain ERapositive, but show reduced expression of the p160 co-activators (SRC1, TIF2 and AIB1) and the co-repressors NCoR and SMRT [31]. The elevated levels of NCoR and SMRT may explain the reduced expression of pS2, CTD and PR in MLET5 cells. The reason for increased NCoR and SMRT proteins in the absence of increases in their mRNA is unclear. However, the ubiquitin ligase mSiah2 has been implicated in regulating proteasomal degradation of NCoR [32]. Siah2 expression has been shown to be estrogen-regulated and to mediate the estrogen-stimulated down-regulation of $\mathrm{NCoR}$, but not SMRT protein [33], although examination of the microarray data did not show a significant difference in Siah2 levels between MCF-7 and MLET5 cells, with 2.3 and 2.1 fold stimulation of Siah2 expression by estrogen, respectively.

High-level of AIB1 expression has been associated with a poor prognosis and non-responsiveness to tamoxifen in ERa-positive breast cancers, with AIB1positive patients also over-expressing HER1, 2 or 3 , being most likely to relapse on tamoxifen [10, 34, 35]. These findings indicate that crosstalk between ERa, AIB1 and growth factor receptor pathways are important in determining response and resistance to tamoxifen. HER2 expression and P-MAPK levels are elevated in the LTED cells [36], similar results being obtained in other LTED cells [37]. However, there was no evidence for increased expression or activity of either EGFR or HER2 in MLET5 cells. Moreover, MAPK phosphorylation levels were comparable in MCF-7 and MLET5 cells, although there was an elevation in PAKT levels and MLET5 cells were about 5 -fold more sensitive to the PI3K inhibitor LY294002 than were MCF-7 cells (Supplementary Figure 2).

\section{MLET5 cells are resistant to apoptosis}

The gene expression microarray analysis showed that genes involved in cell survival and apoptosis are highly differentially regulated in MLET5 cells compared with MCF-7 cells. Examination of the proposed functions of these genes showed that the majority of the anti-apoptotic genes were up-regulated, whilst pro-apoptotic genes were down-regulated in MLET5 cells compared with 
MCF-7 cells. Of particular note were memebers of the Bcl2 gene family, particularly those genes whose expression is estrogen-regulated in MCF-7 cells. Hence, expression of the estrogen-regulated, anti-apoptotic Bcl2 gene was dramatically elevated in MLET5 cells at the mRNA and protein levels, whereas expression of the pro-apoptotic Bcl2 antagonists Bik, Bid, but also BMF and BNIPL was repressed by estrogen and reduced in MLET5 cells. Although expression of the pro-apoptotic BAD gene did not follow this trend, its expression being elevated in MLET5 cells, levels of $\mathrm{Bcl} 2$ expression were increased to a considerably greater extent than those of Bad. It is interesting to note that analysis of the real-time RT-PCR data of $\mathrm{Bcl} 2$ and $\mathrm{Bad}$ expression shows that expression levels were similar in MCF-7 cells in the absence of estrogen, with Bcl2 levels in the presence of estrogen being 2-3 fold greater than levels of Bad. By contrast, in MLET5 cells, Bcl2 levels were 16 and 20 -fold higher in MLET cells compared with MCF-7 cells in the absence and presence of estrogen, respectively. This, taken together with down-regulation of Bid, Bik, as well as BNIPL and BMF, is indicative of anti-apoptotic programming of MLET5 cells, a fact confirmed by the demonstration that MLET5 cells are significantly less sensitive to etoposide-induced apoptosis. Although Bcl2 over-expression has been noted in MCF-7 LTED cells [38], it is not clear if this occurs in the context of other changes in gene expression indicative of overall cell survival, as we have seen in MLET5 cells. Given that Bcl2 positivity and high level Bad expression have been associated with better outcome in breast cancer patients [39, 40], it is likely that the reduced expression of other pro-apoptotic proteins such as Bid and Bik are important for the resistance to apoptotic agents observed in MLET5 cells. Indeed, low Bik expression, as found in MLET5, has previously been associated with resistance to anti-estrogens in MCF-7 cells [41]. Further confirmation of $\mathrm{Bcl} 2$ up-regulation was determined by analysing its expression in LTED cells (Supplementary Figure 5). In these cells there was an increase in $\mathrm{Bcl} 2$ expression as the cells progressed from the quiescent stage (weeks 2-12) to the estrogen hypersensitive stage (weeks 21-40) [28]. As observed for MLET5 cells, Bik and Bid expression fell over this time course, levels of expression in the 
absence of estrogen being reduced to levels similar to, or lower than those seen in the presence of estrogen in MCF-7 cells. Interestingly, Bad expression was unaltered. These findings provide further confirm the association between the development of estrogen independence and altered expression of $\mathrm{Bcl} 2$, Bik and Bid in breast cancer cells.

Taken together, our findings indicate that increased levels of ER $\alpha$ and/or transient over-expression of ERa in breast cancer cells may provide a mechanism for promoting estrogen-independence in breast cancer cells by facilitating cell survival under conditions of low estrogen availability, for example in patients treated with adjuvant aromatase inhibitor therapy. Our data, together with those of other groups who have over-expressed ERa in breast cancer cell lines, would indicate that breast cancer patients with high-level ER expression would respond less well to endocrine treatment. Indeed, other studies have shown that ERa levels are elevated in LTED cells that emerge following longterm growth in estrogen-depleted media. In any case, our data indicate that even a transient over-expression of ERa would be sufficient for the development of resistance to aromatase inhibitors. In summary, the MLET5 line described here provides a useful new model for studying the mechanisms of endocrine response and resistance in breast cancer, and address the paucity of such lines that are currently available. 


\section{Acknowledgments}

We would like to thank Georges Vassaux and Nick Lemoine for facilitating the generation of the adenoviruses used in this study. This work was made possible by grants from Cancer Research UK and the Breast Cancer Research Trust. We are grateful for support from the NIHR Biomedical Research Centre funding scheme. We also thank the CR-UK and the Dept of Health funded Imperial College Experimental Cancer Medicine Centre (ECMC). 


\section{Figure legends}

Figure 1. Transduction of MCF-7 cells with an adenovirus encoding human ERa.

(A) Immunoblots of total cell lysates prepared 48 hours following infection of MCF-7 cells with AdGFP or AdERa at MOI 400. (B) For preparing RNA, estrogen (E2; $10 \mathrm{nM})$ and/or ICl182, $780(\mathrm{ICl} ; 100 \mathrm{nM})$ were added 24 hours prior to harvesting. RT-PCR was performed to analyse expression of the PR, pS2, ERa and GAPDH genes. The expression of each gene was normalised to GAPDH following densitometric analysis and relative expression calculated for each gene, relative to the no ligand control for AdGFP infected cells. The means for the relative expression levels of ERa (C), PR (D) and pS2 (E), as determined from densitometric analysis of RT-PCR carried out on three replicate RNA preparations are shown, the error bars represent the SEM. Significance for gene expression differences was calculated using the t-test. Statistically significant differences $(p<0.05)$ are shown for comparisons for any given treatment between the lines $\left(^{*}\right)$. (F) MCF-7 cells mock infected or infected with AdGFP or AdERa $(\mathrm{MOI}=2,000)$, were grown over a period of 8 months, in estrogendepleted medium. The cell number was determined at monthly intervals. The graphs show the average cell numbers from five independent experiments, the error bars representing the SEM.

\section{Figure 2. The MLET lines grow in an estrogen-independent manner}

MCF-7 (A, C) and MLET5 (B, D) cells were grown in the absence of ligand (NL) or in the presence of increasing concentrations of 17B-estradiol (E2) (A, B). Growth in the presence of anti-estrogens was carried out following the addition of E2 (10 nM), 4-hydroxytamoxifen (OHT; $100 \mathrm{nM}), \mathrm{ICI} 182,780$ (ICl; $100 \mathrm{nM})$ or Raloxifene (RAL; $100 \mathrm{nM}$ ) (C, D). Growth was determined using the SRB assay for three experiments. Error bars depict the standard errors of the mean.

Figure 3. The expression of estrogen-regulated genes in MLET cells 
(A-G) Total RNA prepared from MCF-7 and the MLET lines cultured in estrogendepleted medium, to which $10 \mathrm{nM}$ E2 was added 16 hours prior to harvesting, was subjected to Q-RT-PCR. Expression level for MCF-7 cells in the absence of ligand was taken as 1 , and expression for the other samples are depicted relative to this for three RNA preparations. The grey bars show expression in the absence of estrogen, with the black bars representing expression in the presence of E2. Error bars show the standard errors of the mean. Significance for gene expression differences was calculated using the t-test. Statistically significant differences $(p<0.05)$ are shown for comparisons between the no ligand and estrogen treated samples within each line $\left({ }^{*}\right)$, for the no ligand samples between lines $(\#)$ and for the estrogen-treated samples $(\S)$. $(\mathrm{H})$ Whole cell lysates were immunoblotted, as shown.

Figure 4. Gene expression microarray profiling of MCF-7 and MLET5 cells in the absence and presence of estrogen. (A, B) Venn Diagrams to show the number of genes whose expression was up- or down-regulated $(F C \geq 1.5, p<0.05)$ following the addition of estrogen for 16 hours in MCF-7 and MLET5 cells. (C) A scatterplot is shown for genes whose expression is altered $>2$-fold with estrogen treatment in MCF-7 to show the fold change in expression with estrogen in MLET5 cells, where the estrogen regulation was deemed to be significant $(p<0.05)$. Genes showing a 2 -fold change in MLET5 treated with estrogen are similarly plotted to show genes in MCF-7 cells whose expression was significantly altered in MCF-7 cells following estrogen treatment. (D) The Venn diagram shows the number of differentially regulated genes in MCF-7 and MLET5 cells $(p<0.05, F C \geq 2.0)$.

Figure 5. Expression of genes associated with apoptosis is altered in MLET5 cells. (A-D) Q-RT-PCR for RNA prepared from estrogen-treated MCF-7 and the MLET5 cells. Gene expression is shown relative to the expression in MCF-7 cells in the absence of ligand. Error bars show the standard errors of the mean. Significance for gene expression differences was calculated using the t- 
test. Statistically significant differences $(p<0.05)$ are shown for comparisons between the no ligand and estrogen treated samples within each line $\left({ }^{*}\right)$, for the no ligand samples between lines (\#) and for the estrogen-treated samples (§). (E) MCF-7 and MLET5 cells were cultured in estrogen-depleted medium and whole cell lysates were prepared 24 hours following the addition of $10 \mathrm{nM} \mathrm{E2} \mathrm{(E)} \mathrm{or} \mathrm{an}$ equal volume of ethanol (V).

Figure 6. MLET5 cells are resistant to apoptosis. MCF-7 and MLET cells were treated with etoposide at concentrations ranging from $1 \mathrm{nM}-100 \mu \mathrm{M}$. (A-B) Cell growth was determined using the SRB assay. Shown are the results of three independent experiments. $\mathrm{Gl}_{50}$ values were determined as the concentration of etoposide that inhibited growth by $50 \%$. SD = standard deviation. (C) MCF-7 cells were treated with etoposide at the concentrations shown, or with vehicle (DMSO) for 48 hours, prior to fixation, staining with propidium iodide (PI) and flow cytometric analysis. Shown are representative FACS profiles, with the black line representing the profile obtained for DMSO treated cells and the gray areas represent profiles for etoposide treated cells. (D) Cells treated for 48 hours with DMSO or etoposide were stained with an antibody for Annexin V and with PI. The percentage of cells that stained positive for Annexin $V$ is shown for three independent experiments. Error bars represent the standard errors of the mean (SEM). Statistically significant differences between MCF-7 and MLET5 cells were reached at concentrations of etoposide of 50,75 and $100 \mu \mathrm{M}$ (t-test; $p<0.05$ ). 


\section{References}

1. Osborne, C.K., Tamoxifen in the treatment of breast cancer. N Engl J Med, 1998. 339(22): p. 1609-1618.

2. Ali, S. and R.C. Coombes, Endocrine-responsive breast cancer and strategies for combatting resistance. Nature Rev. Cancer, 2002. 2: p. 101-112.

3. Johnston, S.R. and M. Dowsett, Aromatase inhibitors for breast cancer: lessons from the laboratory. Nat Rev Cancer, 2003. 3(11): p. 821-831.

4. Chen, S., et al., What do we know about the mechanisms of aromatase inhibitor resistance? J Steroid Biochem Mol Biol, 2006. 102(1-5): p. 232-40.

5. Kuukasjarvi, T., et al., Loss of estrogen receptor in recurrent breast cancer is associated with poor response to endocrine therapy. J Clin Oncol, 1996. 14(9): p. 2584-9.

6. Lapidus, R.G., S.J. Nass, and N.E. Davidson, The loss of estrogen and progesterone receptor gene expression in human breast cancer. J Mammary Gland Biol Neoplasia, 1998. 3(1): p. 85-94.

7. Gee, J.M., et al., Phosphorylation of ERK1/2 mitogen-activated protein kinase is associated with poor response to anti-hormonal therapy and decreased patient survival in clinical breast cancer. Int J Cancer, 2001. 95(4): p. 247-254.

8. $\quad$ Kirkegaard, T., et al., AKT activation predicts outcome in breast cancer patients treated with tamoxifen. J Pathol, 2005. 207(2): p. 139-46.

9. Sarwar, N., et al., Phosphorylation of ERalpha at serine 118 in primary breast cancer and in tamoxifen-resistant tumours is indicative of a complex role for ERalpha phosphorylation in breast cancer progression. Endocr Relat Cancer, 2006. 13(3): p. 851-61.

10. Osborne, C.K., et al., Role of the estrogen receptor coactivator AIB1 (SRC-3) and HER-2/neu in tamoxifen resistance in breast cancer. J Natl Cancer Inst, 2003. 95(5): p. 353-61.

11. Jiang, S.Y. and V.C. Jordan, Growth regulation of estrogen receptor-negative breast cancer cells transfected with complementary DNAs for estrogen receptor. $\mathrm{J}$ Natl Cancer Inst, 1992. 84(8): p. 580-91.

12. Zajchowski, D.A., R. Sager, and L. Webster, Estrogen inhibits the growth of estrogen receptor-negative, but not estrogen receptor-positive, human mammary epithelial cells expressing a recombinant estrogen receptor. Cancer Res, 1993. 53(20): p. 5004-11.

13. Lazennec, G. and B.S. Katzenellenbogen, Expression of human estrogen receptor using an efficient adenoviral gene delivery system is able to restore hormonedependent features to estrogen receptor-negative breast carcinoma cells. Mol Cell Endocrinol, 1999. 149(1-2): p. 93-105.

14. Fowler, A.M., et al., Increases in estrogen receptor-alpha concentration in breast cancer cells promote serine 118/104/106-independent AF-1 transactivation and growth in the absence of estrogen. Faseb J, 2004. 18(1): p. 81-93.

15. He, T.C., et al., A simplified system for generating recombinant adenoviruses. Proc Natl Acad Sci U S A, 1998. 95(5): p. 2509-14.

16. Ali, S., et al., The development of a selective cyclin-dependent kinase inhibitor that shows antitumor activity. Cancer Res, 2009. 69(15): p. 6208-15. 
17. Buluwela, L., et al., Inhibiting estrogen responses in breast cancer cells using a fusion protein encoding estrogen receptor-alpha and the transcriptional repressor PLZF. Gene Ther, 2005. 12(5): p. 452-60.

18. Lavinsky, R.M., et al., Diverse signaling pathways modulate nuclear receptor recruitment of $\mathrm{N}-\mathrm{CoR}$ and SMRT complexes. Proc Natl Acad Sci U S A, 1998. 95(6): p. 2920-2925.

19. Shang, Y. and M. Brown, Molecular determinants for the tissue specificity of SERMs. Science, 2002. 295(5564): p. 2465-8.

20. Keeton, E.K. and M. Brown, Cell cycle progression stimulated by tamoxifenbound estrogen receptor-alpha and promoter-specific effects in breast cancer cells deficient in N-CoR and SMRT. Mol Endocrinol, 2005. 19(6): p. 1543-54.

21. Hupperets, P.S., et al., The prognostic significance of steroid receptor activity in tumor tissues of patients with primary breast cancer. Am J Clin Oncol, 1997. 20(6): p. 546-51.

22. Nielsen, K.V., et al., Amplification of ESRI may predict resistance to adjuvant tamoxifen in postmenopausal patients with hormone receptor positive breast cancer. Breast Cancer Res Treat, 2010.

23. Moelans, C.B., et al., Molecular profiling of invasive breast cancer by multiplex ligation-dependent probe amplification-based copy number analysis of tumor suppressor and oncogenes. Mod Pathol, 2010. 23(7): p. 1029-1039.

24. Holst, F., et al., Estrogen receptor alpha (ESR1) gene amplification is frequent in breast cancer. Nat Genet, 2007. 39(5): p. 655-60.

25. Tomita, S., et al., Estrogen receptor alpha gene ESR1 amplification may predict endocrine therapy responsiveness in breast cancer patients. Cancer Sci, 2009. 100(6): p. 1012-7.

26. Albertson, D.G., Conflicting evidence on the frequency of ESR1 amplification in breast cancer. Nat Genet, 2008. 40(7): p. 821-2.

27. Anderson, H., et al., Predictors of response to aromatase inhibitors. J Steroid Biochem Mol Biol, 2007. 106(1-5): p. 49-54.

28. Chan, C.M., et al., Molecular changes associated with the acquisition of oestrogen hypersensitivity in MCF-7 breast cancer cells on long-term oestrogen deprivation. J Steroid Biochem Mol Biol, 2002. 81(4-5): p. 333-41.

29. Lannigan, D.A., Estrogen receptor phosphorylation. Steroids, 2003. 68: p. 1-9.

30. Brunner, N., et al., Acquisition of hormone-independent growth in MCF-7 cells is accompanied by increased expression of estrogen-regulated genes but without detectable DNA amplifications. Cancer Res, 1993. 53(2): p. 283-90.

31. Naughton, C., et al., Progressive loss of estrogen receptor alpha cofactor recruitment in endocrine resistance. Mol Endocrinol, 2007. 21(11): p. 2615-26.

32. Zhang, J., et al., Proteasomal regulation of nuclear receptor corepressormediated repression. Genes Dev, 1998. 12(12): p. 1775-80.

33. Frasor, J., et al., Estrogen down-regulation of the corepressor N-CoR: mechanism and implications for estrogen derepression of $N$-CoR-regulated genes. Proc Natl Acad Sci U S A, 2005. 102(37): p. 13153-7.

34. Shou, J., et al., Mechanisms of tamoxifen resistance: increased estrogen receptorHER2/neu cross-talk in ER/HER2-positive breast cancer. J Natl Cancer Inst, 2004. 96(12): p. 926-35. 
35. Kirkegaard, T., et al., Amplified in breast cancer 1 in human epidermal growth factor receptor - positive tumors of tamoxifen-treated breast cancer patients. Clin Cancer Res, 2007. 13(5): p. 1405-11.

36. Martin, L.A., et al., Enhanced estrogen receptor (ER) alpha, ERBB2, and MAPK signal transduction pathways operate during the adaptation of MCF-7 cells to long term estrogen deprivation. J Biol Chem, 2003. 278(33): p. 30458-68.

37. Santen, R.J., et al., Long-term estradiol deprivation in breast cancer cells upregulates growth factor signaling and enhances estrogen sensitivity. Endocr Relat Cancer, 2005. 12 Suppl 1: p. S61-73.

38. Song, R.X., et al., Down-regulation of Bcl-2 enhances estrogen apoptotic action in long-term estradiol-depleted ER(+) breast cancer cells. Apoptosis, 2005. 10(3): p. 667-78.

39. Callagy, G.M., et al., Bcl-2 is a prognostic marker in breast cancer independently of the Nottingham Prognostic Index. Clin Cancer Res, 2006. 12(8): p. 2468-75.

40. Cannings, E., et al., Bad expression predicts outcome in patients treated with tamoxifen. Breast Cancer Res Treat, 2007. 102(2): p. 173-9.

41. Coser, K.R., et al., Antiestrogen-resistant subclones of MCF-7 human breast cancer cells are derived from a common monoclonal drug-resistant progenitor. Proc Natl Acad Sci U S A, 2009. 106(34): p. 14536-41.

Table 1. Genes associated with cell death showing differential expression in MLET5

Gene

Symbol

MX1

BCL2

IFI6

RTKN

TSPO

BCL6

INHA

BAD

CSE1L

DFFA

PIM1
Description

myxovirus (influenza virus) resistance 1 , interferon-inducible protein p78 (mouse)

B-cell CLL/lymphoma 2

interferon, alpha-inducible protein 6

rhotekin

translocator protein $(18 \mathrm{kDa})$

B-cell CLL/lymphoma 6 (zinc finger protein 51)

inhibin, alpha

BCL2-antagonist of cell death

CSE1 chromosome segregation 1-like (yeast)

DNA fragmentation factor, $45 \mathrm{kDa}$, alpha

polypeptide

pim-1 oncogene
MLET5 vs MCF-7 Vehicle MLET5 E2 vs MCF-7 E2 $\frac{\text { MCF- }}{\text { FC }}$

FC $p$-value $F C$-value $F$

$\begin{array}{lllll}8.9 & 0.000 & 10.8 & 0.000 & 0.6\end{array}$

5.7

0.000

5.8

0.000

5.1

0.000

0.001

0.001

0.000

3.3

0.001

0.000

0.000

2.7

0.000

0.001

0.000

2.2

0.000

0.001

2.1

0.000

0.001

2.1

0.001

0.001

0.010

0.4

1.1

2.3 
NFKBIA

nuclear factor of kappa light polypeptide gene

enhancer in B-cells inhibitor, alpha

0.5

0.001

0.5

0.000

1.0

BIRC3

baculoviral IAP repeat-containing 3

TNF

tumor necrosis factor (TNF superfamily, member 2)

0.5

0.004

0.4

0.001

1.8

BIK

BCL2-interacting killer (apoptosis-inducing)

0.4

0.000

0.4

0.000

1.3

ZAK

sterile alpha motif and leucine zipper containing kinase AZK

$\mathbf{0 . 4} \quad 0.001$

0.4

0.001

0.6

PEA15

phosphoprotein enriched in astrocytes 15

0.4

0.001

0.5

0.002

0.8

SOCS2

suppressor of cytokine signaling 2

$\begin{array}{ll}\mathbf{0 . 4} & 0.000 \\ \mathbf{0 . 2} & 0.000\end{array}$

0.4

0.001

0.4

0.000

1.0

$\mathrm{BCL} 2$ /adenovirus E1B $19 \mathrm{kD}$ interacting protein

like

0.000
0.000

scinderin

0.2

0.2

0.000

0.2

0.000

0.6

SCIN
IGF1R

insulin-like growth factor 1 receptor

ANXA1 annexin $\mathrm{A} 1$

$0.1 \quad 0.000$

0.2

0.000

0.000

1.0

0.1

0.000

0.2

0.6 